\title{
Measuring language lateralisation with different language tasks: a systematic review
}

\author{
Abigail R. Bradshaw ${ }^{\text {Corresp., }}{ }^{1}$ ， Paul A. Thompson ${ }^{1}$ ， Alexander C. Wilson ${ }^{1}$ ， Dorothy V. M. Bishop ${ }^{1}$, Zoe V. J. \\ Woodhead ${ }^{1}$ \\ ${ }^{1}$ Department of Experimental Psychology, University of Oxford, Oxford, United Kingdom \\ Corresponding Author: Abigail R. Bradshaw \\ Email address: abigail.bradshaw@psy.ox.ac.uk
}

Language lateralisation refers to the phenomenon in which one hemisphere (typically the left) shows greater involvement in language functions than the other. Measurement of laterality is of interest both to researchers investigating the neural organisation of the language system and to clinicians needing to establish an individual's hemispheric dominance for language prior to surgery, as in patients with intractable epilepsy. Recently, there has been increasing awareness of the possibility that different language processes may develop hemispheric lateralisation independently, and to varying degrees. However, it is not always clear whether differences in laterality across language tasks with fMRI are reflective of meaningful variation in hemispheric lateralisation, or simply of trivial methodological differences between paradigms. This systematic review aims to assess different language tasks in terms of the strength, reliability and robustness of the laterality measurements they yield with fMRI, to look at variability that is both dependent and independent of aspects of study design, such as the baseline task, region of interest, and modality of the stimuli. Recommendations are made that can be used to guide task design; however, this review predominantly highlights that the current high level of methodological variability in language paradigms prevents conclusions as to how different language functions may lateralise independently. We conclude with suggestions for future research using tasks that engage distinct aspects of language functioning, whilst being closely matched on non-linguistic aspects of task design (e.g. stimuli, task timings etc); such research could produce more reliable and conclusive insights into language lateralisation. This systematic review was registered as a protocol on Open Science Framework: https://osf.io/5vmpt/. 
11 Corresponding author: Abigail Bradshaw, Department of Experimental

12 Psychology, South Parks Road, Oxford, OX1 3UD, 01865271386

13 abigail.bradshaw@psy.ox.ac.uk
Abigail R. Bradshaw ${ }^{1}$

Paul A. Thompson ${ }^{1}$

Alexander C. Wilson ${ }^{1}$

Dorothy V. M. Bishop ${ }^{1}$

Zoe V. J. Woodhead 1

${ }^{1}$ Department of Experimental Psychology, University of Oxford 
14 Abstract: Language lateralisation refers to the greater involvement in language

15 functions of one cerebral hemisphere (typically the left) than the other. Measurement of laterality is of interest both to researchers investigating the neural organisation of the language system and to clinicians needing to establish an individual's hemispheric dominance for language prior to surgery, as in patients with intractable epilepsy.

Recently, there has been increasing awareness of the possibility that different language processes may develop hemispheric lateralisation independently, and to varying degrees. However, it is not always clear whether differences in laterality across language tasks with fMRI are reflective of meaningful variation in hemispheric lateralisation, or simply of trivial methodological differences between paradigms. This systematic review aims to assess different language tasks in terms of the strength, reliability and robustness of the laterality measurements they yield with $\mathrm{fMRI}$, to look at variability that is both dependent and independent of aspects of study design, such as the baseline task, region of interest, and modality of the stimuli. Although we make some recommendations to guide task design, this review predominantly highlights that the current high level of methodological variability in language paradigms prevents conclusions as to how different language functions may lateralise independently. We conclude with suggestions for future research using tasks that engage distinct aspects of language functioning, while being closely matched on non-linguistic aspects of task design (e.g. stimuli, task timings etc); such research could produce more reliable and conclusive insights into language lateralisation. This systematic review was registered as a protocol on Open Science Framework: https://osf.io/5vmpt/. 
It is well established that for most individuals, the left hemisphere is dominant in mediating language functions, as proposed in the $19^{\text {th }}$ century by Paul Broca. Our understanding of such hemispheric specialisation for language in the centuries since still leaves many unanswered questions. Because both expressive and receptive aphasia are so reliably associated with left-hemisphere injury, there tends to be an assumption that left-sided lateralisation is a general feature of language processing, consistent across language domains. Nevertheless, there is evidence that lateralisation may differ within individuals for different language functions, as well as between individuals in side and extent.

Early suggestions of within-individual variability can be found in Rasmussen and Milner's (1975) accounts of Wada testing in patients undergoing surgery to treat epilepsy. They reported on several patients with bilateral speech representation, manifest as a dissociation between the hemispheric organisation of different language functions. Specifically, while anaesthetic injection to one hemisphere selectively disrupted naming and not verbal serial order tasks (e.g. reciting the days of the week), an injection to the other hemisphere produced the reverse pattern. This was construed as evidence that in some cases a 'division of labour' can exist between the hemispheres, in which different 'speech centres' can lateralise to different hemispheres independently. Although such evidence was from the study of a special population, it was argued that such a phenomenon should not necessarily be considered as a result of the type of brain damage and reorganisation that occurs in epilepsy. This raises the possibility that cerebral lateralisation may be a multifactorial rather than a unitary 
58 process, with different language processes developing hemispheric lateralisation

59 independently, and to varying degrees (Bishop, 2013).

60 Indeed, several contemporary models of language predict different patterns of

61 lateralisation for different language processes (Hickok \& Poeppel, 2007; Peelle, 2012;

62 Poeppel, 2014; Price, 2012). These predictions are summarised in Table 1. Different

63 models make different distinctions between language processes and use different

64 terminology, but some general patterns emerge. Acoustic processing of speech input

65 and speech articulation are generally considered to be bilateral, whereas

66 comprehension and generation of more meaningful language is considered to be

67 lateralised. There are some points of disagreement between theories however, either in

68 terms of the extent of lateralisation for a particular language process or the theoretical reasons proposed for such patterns of lateralisation.

Contemporary non-invasive techniques allow more extensive research on patterns of laterality than earlier clinical studies. Functional magnetic resonance imaging (fMRI) data can be used to calculate a laterality index (LI), a single value description of the predominance of activity in one hemisphere. The $\mathrm{LI}$ is calculated as the difference between activity in each hemisphere ( $L$ and $R$ ) divided by the total activity across the hemispheres.

$$
L I=\frac{L-R}{L+R}
$$
appears to support the notion that language laterality is not unitary, because we can see differences between tasks in the strength of the laterality measurements they yield. 
80 However, the reasons for such variability in LI strength across language tasks can be

81 debated; could it simply be an artefact of more trivial differences in task design, or does

82 it reveal something fundamental about the hemispheric organisation of different

83 components of language? Of course, trying to devise tasks so as to equate diverse

84

85

language functions such as speech production and speech comprehension is an unrealistic and inappropriate goal. However, more can be done to optimize protocols for LI measurement, in order to try to reduce the possibility of differences in task sensitivity or measurement error being responsible for variability in Lls across tasks.

This systematic review aims to assess evidence on the robustness of laterality measured using fMRI with different language tasks, from studies published between 2000 and 2016. This is done with a view to providing some guidance on optimizing variables such as region of interest and baseline task on a task-by-task basis. Such optimization will be important before tasks can be used to systematically probe patterns of co-lateralisation and independent lateralisation of different language functions. We hypothesise that 1) different language tasks will demonstrate different levels of lateralisation and 2) parameters such as the region of interest and baseline task used will have effects on laterality measurement that may be task-specific.

\section{Materials and Methods}

A protocol for this systematic review has been registered on Open Science Framework and can be found at https://osf.io/5vmpt/. We do not cover here generic issues such as how thresholding and other methodological issues affect laterality measurement, since these were the focus of a companion review based on the same source material (Bradshaw, Bishop, \& Woodhead, 2017). 


\section{Eligibility criteria}

104

105

106

107

108

109

110

111

112

113

114

115

116

117

118

119

120

121

122

123

124

We selected papers published between 2000 and 2016 that used fMRI to study

language lateralisation and that met the following criteria: (1) the paper reported LIs for language calculated using fMRI; (2) the paper studied healthy monolingual adults; and

(3) if both patients and healthy controls were studied, the data for controls were reported separately. Papers were excluded if: (1) they exclusively studied structural asymmetries, children or bilingualism; or (2) they used language tasks with nonEuropean languages. The search was restricted to studies of healthy, monolingual, adult participants to reduce heterogeneity within our study sample.

\section{Search strategy and selection process}

The following search terms were used to search papers published between 2000 and 2016 in Web of Science: laterali* OR asymmetr* OR dominance; AND language OR reading; AND fMRI OR functional MRI OR functional magnetic resonance imaging OR functional MR OR function MRI; NOT schizophrenia; NOT development*; NOT child*; NOT bilingual ${ }^{*}$. This was last searched on $05 / 12 / 16$. Titles and abstracts of the resulting 90 papers were then screened by two of the review authors (Abigail Bradshaw and Zoe Woodhead), followed by full-text scans to determine whether the inclusion criteria were met. Selected lists were compared between reviewers and any discrepancies discussed and a mutual decision made. This resulted in the selection of 34 papers. We next screened papers citing these 34 articles. A further 50 articles were identified as meeting our criteria, yielding a total of 84 papers. A final check of papers led to the discounting of 7 papers deemed to not sufficiently meet criteria, with a further paper being 
125 discounted during conductance of the review. The full search and selection process is

126 illustrated in in Fig. 1. A list of the 76 selected papers is given in Appendix S1.

\section{Data collection and analysis}

128 Information on variables of interest for each study were collected and managed using

129 REDCap electronic data capture tools (Harris et al., 2009) hosted at Oxford University.

130 REDCap (Research Electronic Data Capture) is a secure, web-based application

131 designed to support data capture for research studies, providing: 1) an intuitive interface

132 for validated data entry; 2) audit trails for tracking data manipulation and export

133 procedures; 3 ) automated export procedures for seamless data downloads to common

134 statistical packages; and 4) procedures for importing data from external sources. The

135 full database can be found in Appendix S2. A summary table drawn from this database

136 with the key outcomes of interest for this paper is provided in Appendix S3. For each

137 paper, we recorded: sample size and handedness, the type of fMRI design used, the

138 activity measures used for LI calculation, the threshold level chosen, the use of global or

139 regional LI calculation, the specific regions considered, the language and baseline tasks

140 used, the use of a single or a combined task analysis and the task difficulty.

141 The variable nature of the methods and measures reported by different papers did

142 not permit performance of a meta-analysis. Instead, to illustrate the strength of laterality

143 measured across different language tasks, we produced forest plots showing the mean

144 and $95 \%$ confidence intervals of LI values reported in the studies, as well as their

145 associated methods of LI calculation, region(s) of interest, and language and baseline

146 tasks (Fig. 2, 3 and 4). These outcome measures were not always available in every

147 paper however; LI values and/or their spread were sometimes omitted altogether or 
148 given in a different form e.g. median values. Where standard deviation or standard error

149 were given, these were converted to $95 \%$ confidence intervals. A spreadsheet of the

150 data that was used to generate these forest plots in given in Appendix S4.

151 To avoid the potential confound of heterogeneity in samples in terms of

152 handedness, these forest plots only included mean Lls reported by our selected studies

153 measured either from right handed participants, or from mixed handedness samples

154 where the relative proportion of left and right handers was representative of the general

155 population (around 10\% left handed, $90 \%$ right handed). We excluded LIs reported from

156 studies that selected a participant group on the basis of their pre-known lateralisation.

157 Where more than one frontal LI was reported from a study, inferior frontal gyrus (IFG)

158 LIs were selected; where more than one temporoparietal LI was reported, the LI

159 calculated from the largest area of temporoparietal cortex was selected. Forest plots

160 were created using a script in $\mathrm{R}$, which is available along with the data on open science

161 framework (https://osf.io/7s4hv/).

162 Results

163 The main language tasks identified in our search are listed in Table 2, with counts of the 164 number of studies using each task (one study is missing from these counts as their 165 language task did not fit in to any of these categories). Mean Lls reported from studies 166 using these different language tasks are given in Fig. 2, 3 and 4. A single language task

167 typically engages multiple language processes in an overlapping fashion. This may 168 either be because of task requirements, or reflect spontaneous engagement of task 169 irrelevant processing by the perception of linguistic stimuli. Table 2 provides one 170 characterisation of the different language processes engaged by each of the language 
171 and baseline tasks included in this review. Comparing the language processes engaged

172 by active and baseline tasks is crucial, because when activation for a language task is

173 subtracted from a baseline the aim is to isolate specific linguistic functions, and the

174 extent to which this is successful will depend on the demands of the baseline task.

In the following review, we discuss each language task in turn, with reference to

176 the involvement of different language processes and the forest plots of mean LI values

177 (Fig. 2, 3 and 4). Table 2 highlights the difficulty in designing a task which isolates a

178 single language function in order to study its laterality; this must be kept in mind when

179 interpreting LI values and theorising on the lateralisation of particular language

180 processes.

181

182

183

184

185

186

187

188

189

190

191

192

\section{Verbal fluency}

Verbal fluency tasks have traditionally been viewed as the gold standard for measuring language lateralisation with fMRI. Here, the participant must generate (covertly or overtly) words that meet certain criteria, such as beginning with a particular letter (phonemic fluency), belonging to a particular semantic category (semantic fluency), verbs that are semantically associated with a particular noun (verb generation), or words that are antonyms/synonyms (antonym/synonym generation). Any lateralisation induced by this task may thus reflect a mixture of phonological, semantic, word retrieval and speech motor planning/articulation processes (see Table 2). Lateralisation of speech motor processes is a subject of debate (see Table 1), with some considering them left lateralised (Hickok \& Poeppel, 2007), but others bilateral (Poeppel, 2014; Price, 2012). 
Across the papers reviewed here, verbal fluency tasks are consistently reported as yielding the strongest laterality when compared to other receptive and expressive tasks within studies (Baciu et al., 2005; Deblaere et al., 2002; der Haegen, Cai, \& Brysbaert, 2012; Dodoo-Schittko, Rosengarth, Doenitz, \& Greenlee, 2012; Harrington, Buonocore, \& Farias, 2006; Jensen-Kondering, Ghobadi, Wolff, Jansen, \& Ulmer, 2012; Niskanen et al., 2012; Ocklenburg, Hugdahl, \& Westerhausen, 2013; Ramsey, Sommer, Rutten, \& Kahn, 2001; Vikingstad, George, Johnson, \& Cao, 2000; Zaca, Jarso, \& Pillai, 2013a). Studies included in the forest plots produced here report a wide spread of LI values for verbal fluency tasks, ranging from 0.05 to 0.94 (see Fig. 2).

A number of studies have compared the use of different verbal fluency paradigms. When a frontal region of interest $(\mathrm{ROI})$ is used, semantic fluency is reported as less strongly lateralising than verb generation or phonemic fluency (Kleinhans, Mueller, Cohen, \& Courchesne, 2008; Ruff et al., 2008; Sanjuan, Bustamante, et al., 2010). Conversely, two studies using combined frontal and temporoparietal ROls reported no differences in their strength of laterality (Rutten, Ramsey, van Rijen, \& van Veelen, 2002; Tailby, Weintrob, Saling, Fitzgerald, \& Jackson, 2014). This suggests that these tasks may differ in the extent of lateralisation they induce across different language areas (see following section, effect of region of interest). Interestingly, multiple studies report that LIs from generation tasks can vary substantially depending on methodological choices made when calculating laterality, such as the threshold chosen

214 (Dodoo-Schittko et al., 2012), the use of normalisation, smoothing and clustering techniques (Baciu et al., 2005), and the activity measure used (Harrington et al., 2006). 
217 Verbal fluency tasks tend to induce the strongest laterality in frontal ROIs (Gaillard et

218 al., 2003; Niskanen et al., 2012; Ocklenburg et al., 2013; Partovi et al., 2012a; Partovi et

219 al., 2012b; Propper et al., 2010; Szaflarski et al., 2008; Vernooij et al., 2007; Vikingstad

220 et al., 2000; Vingerhoets et al., 2013; Zaca, Jarso, \& Pillai, 2013). Although they can

221 induce strong laterality in temporoparietal ROls (Harrington et al., 2006; Jensen-

222 Kondering et al., 2012; Stippich et al., 2003), this may not be significantly greater than

223 other tasks within this ROI (Zaca et al., 2013). However, this may depend on the

224 particular fluency task used; Jensen-Kondering et al. (2012) reported that while

225 phonemic fluency and verb generation yielded the strongest lateralisation for a frontal

$226 \mathrm{ROI}$, the strongest laterality for a temporoparietal ROI was seen with semantic fluency,

227 consistent with a role for such areas in semantic cognition.

228 Effect of baseline task

229 Although the majority of studies using verbal fluency tasks employed a passive baseline

230 task such as fixation, a number used active baselines such as finger tapping or silent

231 word repetition (see Fig. 2 and Table 2). Dodoo-Schittko et al. (2012) reported that an

232 active baseline task which required subvocal manipulation of the order of syllables

233 within a pseudoword yielded significantly stronger laterality for a verb generation task

234 compared to the use of a passive resting baseline. This is consistent with the idea of

235 subtraction of bilateral activity related to speech-motor planning (Poeppel, 2014; Price, 236 2012).

\section{Sentence generation}


238 Sentence generation requires participants to generate sentences to describe presented

239 pictures. These sentences may either be pre-defined and learnt prior to scanning, or

240 generated during the scan itself. Relative to word generation, additional syntactic and

241 semantic-integration processes are involved in the construction of a sentence (see

242 Table 2). These are argued to be left lateralised by multiple models (Peelle, 2012;

243 Poeppel, 2014). Poeppel's (2014) COM-PRE hypothesis makes a distinction between

244 bilateral processing within input and output interfaces (e.g. auditory perception and

245 speech production), and left dominant processing of combinatorics and composition

246 (COM) or linguistically-based predictions (PRE). Similarly, Peelle (2012) predicts that

247 while unconnected language (e.g. single words) is processed bilaterally, processing of

248 connected language that requires more complex linguistic operations is left lateralised.

249 Thus, these models might predict stronger laterality for sentence generation over word

250 generation paradigms, due to the additional sentential processing demands.

251

252

253

254

255

256

257

258

259

260

\section{LI strength, reliability and robustness}

Mean LIs reported from sentence generation studies are illustrated in Fig. 3. High mean

LIs of between 0.74 and 0.89 have been reported for sentence generation, both when sentences are pre-learnt prior to scanning (e.g. Stippich et al., 2003), and when they are actively generated during the scan (e.g. Tzourio-Mazoyer, Joliot, Marie, \& Mazoyer, 2016). However, other studies have reported more modest laterality estimates of between 0.48 and 0.65 , again with both variants of the task (Mazoyer et al., 2014;

Partovi et al., 2012a; Partovi et al., 2012b). Thus, it does not appear to be the case that strength of laterality differs according to whether sentences are generated spontaneously during the scanning session, or learnt prior to scanning. Two studies 
261 within our search measured laterality for semantic fluency and sentence generation

262 within the same participants (Partovi et al., 2012a; Stippich et al., 2003); however, these

263 studies reported differences in strength of laterality between the tasks in different

264 directions. Further, they used the version of the sentence generation task in which

265 sentences are learnt prior to scanning, so task demands were not well matched. Thus,

266 there is currently insufficient data with these tasks to evaluate predictions of stronger

267 laterality for sentence over word processing. Partovi et al., (2012a) report good

268 reproducibility for sentence generation, however their analysis simply looked at

269 significant differences in group means over repeated testing, and not at reproducibility

270 of individual participant's LIs.

271 Effect of ROI

272 There is mixed evidence as to whether sentence generation yields differences in the

273 laterality measured from frontal versus temporoparietal ROIs. While Partovi et al.

274 (2012a; 2012b) and Stippich et al. (2003) reported equivalent strength of laterality

275 across both, Tzourio-Mazoyer et al. (2016) found significantly stronger laterality in

276 frontal than temporal areas. Interestingly, in contrast to the exclusively right handed

277 samples of the former studies, this latter study used a mixed handedness sample, with

278 an overrepresentation of left handers. This suggests the possibility that greater regional

279 heterogeneity may characterise the atypical profiles of language lateralisation that are

280 more often found within atypical handedness samples.

\section{Effect of baseline}


282 When sentences are learnt prior to scanning, studies generally employ simple cross-

283 fixation or rest as a baseline (Partovi et al., 2012a; Partovi et al., 2012b; Stippich et al.,

284 2003). Conversely, the two studies using spontaneous generation of sentences during

285 the scanning session used an active linguistic baseline, in which participants covertly

286 generated the months of the year (Mazoyer et al., 2014; Nathalie Tzourio-Mazoyer et

287 al., 2016). A comparison of these baselines in terms of the language processes isolated

288 by each contrast is given in Table 3 . As can be seen, the active baseline subtracts out

289 activity related to speech motor planning to leave those processes specific to the

290 construction of novel sentences, such as syntactic and lexico-semantic processing;

291 conversely, the contrast with rest results in poor isolation of such language processes.

292 This highlights the need to consider carefully the functions one wishes to isolate when

293 choosing a suitable baseline, and the implications this will have for interpretation of

294 measured laterality in relation to linguistic processes.

295

296

297

298

299

300

301

302

303

\section{Passive speech listening}

Passive speech listening as a language paradigm appears to yield more variable laterality estimates, perhaps reflective of the wide variety of language processes that it can engage (see Table 2). Lower-level acoustic processing of speech sensory input is predicted to be bilateral by multiple models (Hickok \& Poeppel, 2007; Peelle, 2012;

Poeppel, 2014; Price, 2012). However, there are discrepancies in the extent of lateralisation assumed for mapping of sound to meaning, considered bilateral by Hickok and Poeppel (2007) but left lateralised by other authors (Peelle, 2012; Poeppel, 2014; Price, 2012) owing to the need to process meaning at a sentential level. Thus, 
304 depending on the baseline subtraction used, different levels of processing may be

305 isolated to result in variable levels of laterality.

306

307

308

309

310

311

312

313

314

315

316

317

318

319

320

321

322

323

324

\section{LI strength, reliability and robustness}

The majority of studies using passive listening tasks reported very weak average LIs (see Fig. 4 for mean LI values), indicating bilateral activation; indeed, passive listening is often the most weakly lateralising task when compared to others (Binder, Swanson, Hammeke, \& Sabsevitz, 2008; Harrington et al., 2006; Miro et al., 2014; Ocklenburg et al., 2013; N Tzourio-Mazoyer et al., 2015). A notable exception to this was presented by Thivard et al. (2005) who reported a mean laterality index of 0.72 within a frontal $\mathrm{ROI}$ for a passive story listening task, stronger than that seen in this ROI for a semantic fluency task (0.51). We also note that high test-retest correlations have been reported for speech listening (Razafimandimby et al., 2007).

\section{Effect of ROI}

Studies are inconsistent as to whether stronger laterality is found for speech listening within a frontal or a temporoparietal ROI. Harrington et al. (2006) reported that temporoparietal Lls were stronger and more reliable than frontal LIs, whereas other studies have reported weaker and more variable LIs for a temporal compared to a frontal ROI (Miro et al., 2014; Ocklenburg et al., 2013; Thivard et al., 2005). In general, posterior language areas appear to be poorly lateralised for receptive speech listening tasks, although this may depend on the baseline task employed (see paragraph below).

Effect of baseline task 
325 The varying levels of asymmetry reported for speech listening tasks may in part be

326 attributable to the baseline used by different studies. Binder et al. (2008) found that

327 changing the baseline from rest to tone listening raised the average $\mathrm{LI}$ for word listening

328 from 0.1 to 0.52 . In this regard it is interesting that the two studies reporting near-zero

329 average $\mathrm{LI}$ values for speech listening employed rest as a baseline task (Miro et al.,

330 2014; Ocklenburg et al., 2013). Conversely, Thivard et al. (2005) and Harrington et al.

331 (2006) both used backwards speech listening as a baseline and reported stronger

332 laterality measurements for speech listening. This effect of baseline is consistent with

333 the idea of bilateral early auditory processing that must be subtracted out by a non-

334 linguistic auditory stimulus in order to reveal asymmetry for higher-level 'central

335 language processes' (Peelle, 2012; Poeppel, 2014; Price, 2012).

\section{Text reading}

337 Reading text or narrative requires decoding of orthography into phonological representations, semantic and syntactic processing of the decoded sentence, and binding within and across sentences to arrive at an overall understanding of text meaning (see Table 2). Visual word form processing is considered to rely on a

341 lateralised ventral occipito-temporal region, although this may not reflect a left

342 specialisation for orthography per se (Price, 2012).

\section{LI strength and effect of ROI}

Our search identified only two papers investigating lateralisation of text reading. Both studies used the same covert (silent) text reading task with a baseline of covert reading

346 of text composed of pronounceable non-words. Backes et al. (2005) reported 
347 moderately strong laterality $(\mathrm{LI}=0.59)$ using a combined frontal-temporoparietal ROI,

348 whereas (Deblaere et al., 2002) reported weak laterality $(\mathrm{LI}=0.21)$ using a global $\mathrm{LI}$

349 (see Fig. 4). This supports the hypothesis that global LIs are generally weaker than

350 regional LIs.

\section{Phonemic judgement}

352 Phonemic judgement tasks require a decision relating to phonological structure; most

353 commonly, a rhyme judgement. This task relies on mapping of acoustic or visual input

354 onto phonological units such as phonemes and syllables, a process known as decoding.

355 The precise nature of these stored phonological codes remains a debate; according to

356 theorists in the tradition of the motor theory of speech perception (e.g. Liberman \&

357 Mattingly, 1985), these are represented as speech motor gestures in left premotor

358 cortex. Hickok and Poeppel (2007) argue that while the phonological codes themselves

359 are bilaterally represented, the process of their mapping onto articulatory motor

360 representations relies on a left lateralised dorsal stream. Conversely, other models

361 propose that such processing of single words is a less strongly lateralised process

362 (Peelle, 2012).

363

364

365

366

367

368

\section{LI strength, reliability and robustness}

Phonemic judgement tasks yield relatively strong laterality, with reported LI values ranging from 0.41 to 0.84 (see Fig. 3). However, when compared to other tasks, phonemic judgement is often reported as more weakly lateralising (Baciu et al., 2005; Niskanen et al., 2012; Seghier et al., 2004). Phonemic judgement may be superior to other tasks however in terms of robustness and reproducibility. Morrison et al. (2016) 
369 reported that a rhyming decision task demonstrated greater reliability than a word

370 generation task, yielding reproducible dominance classifications in $100 \%$ of participants,

371 and average test-retest correlations for LI values of 0.9 and above. Furthermore, such

372 reproducibility of Lls obtained with rhyming decision was more robust against changes

373 in the activity measure used for LI calculation.

374 Effect of ROI

375 Rhyming decision tasks yield particularly strong laterality when a frontal or a combined

376 frontal-temporoparietal ROI is used (Baciu et al., 2005; Clements et al., 2006; Cousin et

377 al., 2007; Niskanen et al., 2012). For example, Cousin et al. (2007) identified a

378 particularly strong leftward asymmetry for the inferior frontal gyrus during rhyme

379 detection. Thus, frontal ROls may be optimal for yielding the strongest laterality with this 380 task.

381 Effect of baseline task

382 All studies within our search using phonemic judgement were found to employ an active 383 perceptual decision baseline task on either non-linguistic material (e.g. line orientation 384 matching) or nonsense words or characters (e.g. nonsense word font matching). This 385 subtracts out non-linguistic working memory processes (see Table 2), as well as basic 386 visual processing.

\section{Semantic decision}

Semantic decision tasks require a judgement about a word's semantic content or about the semantic relationship between a pair of words, such as whether two words belong to 390 the same category. Such conceptual knowledge is proposed to rely on a distributed 
391 processing network, with different brain areas each contributing to different aspects of

392 an item's representation (Warrington \& McCarthy, 1983; 1987; Warrington \& Shallice,

393 1984). In addition to this distributed network, Patterson, Nestor, and Rogers (2007)

394 have argued for the existence of a 'semantic hub' within the bilateral anterior temporal

395 lobes that integrates the distributed modality-specific representations into one amodal

396 representation. However, a recent meta-analysis of functional imaging studies by Rice,

397 Ralph and Hoffman (2015) suggested that while conceptual knowledge does appear to

398 be represented bilaterally in the anterior temporal lobes, left lateralised activity was

399 more likely when semantic content was accessed linguistically. This is in contrast to the

400 predictions of Hickok \& Poeppel's (2007) model of language in which access to lexico-

401 semantics from speech processing (via the ventral stream) is considered as a bilateral

402 process.

403 LI strength, reproducibility and robustness

404 The strength of laterality reported for semantic decision tasks is quite variable, ranging

405 from near-zero to around 0.8 (see Fig. 4 for mean LI values). This may depend on the

406 type of semantic decision required. Tasks which require judgement of the semantic

407 relatedness of two words appear to yield relatively strong laterality, ranging from 0.59 to

4080.84 (Bethmann, Tempelmann, Bleser, Scheich, \& Brechmann, 2007; Fernandez et al.,

409 2001; Häberling, Steinemann, \& Corballis, 2016; Seghier et al., 2004). In contrast,

410 category membership tasks with single words appear to give much lower LIs, ranging

411 from 0.03 to 0.52 (Deblaere et al., 2002; Hund-Georgiadis, Lex, Friederici, \& von

412 Cramon, 2002; Hund-Georgiadis, Lex, \& von Cramon, 2001; Ramsey et al., 2001; van

413 Oers et al., 2010). This suggests that it may be the process of integrating and 
414 comparing across semantic representations for different concepts that is strongly

415 lateralising; conversely a simple lexical look-up to determine the category membership

416 of a single concept may not be strongly lateralised.

417 Jansen et al. (2006) reported very low reproducibility for synonym decision LIs

418 across a range of different $\mathrm{LI}$ calculation methods within Broca's area; much higher

419 reproducibility was found however when a temporoparietal ROI was used. Conversely,

420 Harrington et al. (2006) reported high test-retest correlations for an abstract/concrete

421 semantic decision task across both frontal (IFG) and temporoparietal ROIs. This

422 discrepancy between the two studies could be due to the differences in the tasks they

423 used.

424 Effect of ROI

425 The majority of studies report no significant differences in the magnitude of laterality

426 found within temporoparietal and frontal ROIs for semantic decision tasks (Bethmann et

427 al., 2007; Häberling et al., 2016; Harrington et al., 2006; Hund-Georgiadis et al., 2002;

428 Ramsey et al., 2001; van Oers et al., 2010). Some studies have reported differences

429 across ROls, however these can be in opposite directions (Fernandez et al., 2001;

430 Szaflarski et al., 2008). As discussed, some evidence suggests that Lls calculated from

431 temporoparietal ROIs for semantic decision may be more reproducible than those

432 calculated from frontal ROIs (Jansen et al., 2006).

433 Effect of baseline tasks

434 Semantic decision laterality is also strongly influenced by the baseline task used. Binder

435 et al. (2008) and Hund-Georgiadis et al. (2002, 2001) manipulated the baseline and 
436 found that the use of an active perceptual decision task as opposed to passive rest

437 yielded a large increase in the strength and consistency of semantic decision laterality.

438 Binder et al. (2008) argued that resting baselines are unsuitable for subtraction with

439 semantic decision, since they allow for the activation of conceptual language

440 representations as the participant 'day dreams' and engages in 'inner speech'. An active

441 perceptual decision baseline interrupts such ongoing conceptual processing and

442 engages the same executive and attentional processes as the language paradigm. This

443 subtraction is shown in Table 4, which illustrates the better isolation of semantic

444 processes that this baseline provides compared to the contrast with rest. Baseline tasks

445 that engage linguistic processing themselves may result in reduced laterality; for

446 example, Deblaere et al. (2002) suggested their finding of weak laterality for semantic

447 decision may have been due to a vowel decision baseline task. However, it should be

448 noted that Binder et al. (2008) reported identical laterality strength (a mean LI of 0.62 )

449 for semantic decision using either a baseline of tone decision or phoneme decision.

450 Overall, this evidence suggests that baseline tasks used for semantic decision must be

451 active, sufficiently engaging and challenging so as to prevent 'day-dreaming', and

452 ideally involve material from a non-linguistic domain e.g. symbols or tones.

\section{Sentence comprehension}

454 Sentence comprehension tasks require some judgement about the content of a spoken

455 or written sentence. Syntactic and semantic processing are often confounded (see

456 Table 2); for example, the task may require participants to decide if two sentences with

457 different grammatical constructions have the same meaning. However, they are

458 noteworthy among other tasks in the extent of their syntactic processing requirements. 
459 Laterality of syntax has been a subject of debate, with some authors arguing for a

460 bilateral involvement in syntax (e.g. Hund-Georgiadis et al., 2001), but others arguing

461 for a left dominance (Friederici, 2011; Tyler et al., 2011; Wright, Stamatakis, \& Tyler,

462 2012). At a more general level, multiple models would predict left lateralisation for the

463 sentence-level processing engaged by this task, without making specific claims about

464 lateralisation of syntactic processing per se (e.g. Peelle, 2012; Poeppel, 2014; Price, 465 2012).

$466 \quad$ LI strength, reliability and robustness

467 Multiple studies report strong laterality for sentence comprehension tasks (Harrington et 468 al., 2006; Jensen-Kondering et al., 2012; Kennan, Kim, Maki, Koizumi, \& Constable, 469 2002; Niskanen et al., 2012; Sanjuan, Forn, et al., 2010; Vassal et al., 2016), with LI 470 values ranging from 0.55 to 0.88 (see Fig. 3). Studies which compare sentence 471 comprehension laterality measures to those of other tasks suggest that it can 472 outperform semantic decision, phoneme decision, story listening and naming tasks in 473 terms of the strength of laterality, although this can depend on the ROI (Harrington et 474 al., 2006; Niskanen et al., 2012).

475 Effect of ROI

476 Evidence appears inconsistent as to the effect of ROI on the laterality obtained with sentence comprehension tasks. Studies have reported both stronger laterality for frontal 478 than temporoparietal ROIs (Jensen-Kondering et al., 2012; Niskanen et al., 2012) and 479 vice versa (Harrington et al., 2006; Sanjuan, Forn, et al., 2010). In terms of reliability of 480 lateralisation, Harrington et al (2006) reported very high reproducibility for a visual 
481 sentence comprehension task across both frontal and temporoparietal ROIs, with test-

482 retest correlations above 0.9 . In contrast, auditory sentence comprehension yielded

483 more reliable lateralisation within a temporoparietal than a frontal ROI. Modality of the

484 stimuli may thus affect which ROI is optimal.

485 Effect of baseline task

486 Generally, active baselines are employed for sentence comprehension tasks. A notable 487 exception is seen in Harrington et al. (2006) who used passive listening to backwards 488 speech as a baseline for auditory sentence comprehension. This passive baseline may 489 explain the weaker laterality they reported as compared to other studies (mean $\mathrm{LI}$ of 490 around 0.45). Interestingly, Sanjuan et al. (2010) who also reported a relatively low level 491 of laterality compared to other studies used phoneme decision as a baseline. As 492 previously discussed in relation to a study by Deblaere et al. (2002) using semantic 493 decision, it is possible that the use of such a baseline with high linguistic processing 494 demands may lower the strength of the laterality seen.

\section{Naming}

496 Naming tasks require the generation of the name of an item in response to either a 497 visual (pictorial) or verbal description. According to Hillis' (2007) model of naming, 498 picture naming involves three major levels of processing; a semantic level in which 499 amodal general and specific semantic information is accessed from a structural

500 description of an object; a lemma level which involves the defining features of an object 501 at a more abstract level (e.g. what makes a sheep a sheep); and a

502 phonological/orthographical level, in which the phonological and orthographical 
503 representations associated with that concept are accessed. Thus, naming tasks have

504 the potential to engage multiple key components of the language network (see Table 2).

505 LI strength, reliability and robustness

506 Studies using naming as a language activation task report a wide variety of LI values,

507 ranging from 0.08 to 0.96 (see Fig. 3). This can partly be explained by variation in the

508 nature of the naming task used. Zero to moderate laterality has been reported for

509 picture naming tasks, which are often the least lateralising when compared to other

510 tasks (Deblaere et al., 2002; Harrington et al., 2006; Jansen et al., 2006; van Oers et

511 al., 2010; Vikingstad et al., 2000). However, the lateralising ability of naming tasks can

512 be increased by the addition of sentence comprehension demands. A naming from

513 written or auditory description task known as 'responsive naming' requires

514 comprehension of a question describing an object in order to generate the required

515 name. This has been reported to yield strong laterality across both frontal and temporal

516 ROls, in the range of 0.65 to 0.96 (W D Gaillard et al., 2002; Niskanen et al., 2012).

517 This increase in laterality with the addition of sentence-level processing is consistent

518 with models of language which predict an increase in laterality for connected versus

519 unconnected language i.e. structured sentences versus single words (Peelle, 2012;

520 Poeppel, 2014).

521 Picture naming also shows poor reliability in laterality measurement. Jansen et al.

522 (2006) reported that picture naming did not determine dominance reproducibly in about

523 a third of participants. Rutten et al. (2002) similarly reported a failure to find significant

524 test-retest correlations for naming LIs. Significant test-retest correlations were reported

525 by Harrington et al. (2006) for a picture naming task at around the same level as those 
526 seen for semantic decision; however, reproducibility of naming laterality was lower than

527 that seen for verb generation and sentence comprehension.

528 Effect of ROI

529 Naming tasks do not appear to favour one ROI over another in laterality measurement.

530 We found two studies which reported differences in the laterality measured from frontal

531 and temporoparietal ROls, however this difference was in opposite directions

532 (Harrington et al., 2006; Brennan et al., 2007). The majority of studies instead report

533 highly similar strength of laterality across frontal and temporoparietal ROls (Gaillard et

534 al., 2002; Niskanen et al., 2012; van Oers et al., 2010; Vikingstad et al., 2000).

535 Furthermore, Rutten et al. (2002) reported similar levels of reproducibility of naming LIs

536 for both regions.

\section{Effect of baseline task}

538 The baselines used with picture naming provide interesting evidence on the processes 539 underlying its laterality. Deblaere et al. (2002) reported near zero laterality for picture

540 naming using a baseline which required participants to name the position of the

541 intersection of four lines (e.g. up, down, left, right). This task involves engagement in

542 similar semantic and word retrieval processes (see Table 2), which may explain the

543 weak laterality measured. However, Brennan et al. (2007) reported strong laterality for a

544 picture naming task with a number counting baseline. This would subtract out speech

545 production and word retrieval processes for an automated speech sequence, predicted

546 to involve bilateral activity (Price, 2012; Poeppel, 2014). This subtraction would thus 
547 isolate spontaneous non-automated retrieval and word generation processes which may

548 engage left hemisphere language systems, increasing measured laterality.

\section{Combined Task Analysis}

550 Combined task analysis (CTA) involves the calculation of Lls from contrast images

551 generated by combining scans across multiple language tasks. This method identifies

552 commonalities between tasks' activity patterns in order to isolate the 'core' language

553 network, and exclude task-specific, non-linguistic activity caused by differences in task

554 design that may influence the LI value. In this way, CTA can represent a theoretical

555 alternative to baseline tasks to subtract domain-general activity, assuming that different

556 tasks involve different patterns of non-linguistically relevant activity. Indeed, there is

557 evidence that CTA results in higher and more reliable and robust estimates of laterality

558 for language (Dodoo-Schittko et al., 2012; Harrington et al., 2006; Jansen et al., 2006;

559 Niskanen et al., 2012; Ramsey et al., 2001; Rutten et al., 2002; Sommer, Ramsey,

560 Mandl, \& Kahn, 2003; van Rijn et al., 2008).

561

Nevertheless, the theoretical assumptions which motivate CTA can be

562 questioned. CTA assumes that variability in laterality for different tasks should be

563 ascribed to non-linguistic processes or viewed simply as measurement error, rather

564 than reflecting the underlying nature of hemispheric organisation for language (e.g.

565 Ramsey et al., 2001). Such a theoretical stance ignores the possibility of

566 multidimensional lateralisation across different language processes. Indeed, recent

567 fMRI studies have reported cases of dissociated laterality for different language

568 functions within individuals (der Haegen et al., 2012; Häberling et al., 2016; Vikingstad

569 et al., 2000; see Bradshaw et al., 2017 for a review), corroborating those early clinical 
570 reports of a 'division of labour' across the hemispheres in patients with bilateral

571 language representation (Rasmussen \& Milner, 1975). It is not yet known whether such

572 crossed language dominance has significant functional implications for language

573 abilities. Bishop (2013) speculated that having expressive and receptive language

574 functions in opposite hemispheres may make one more vulnerable to development of

575 language disorders or impairments.

576 Such differences in dominance between language tasks would be lost in a CTA,

577 since combining scans across paradigms would result in few areas of common

578 activation and thus a loss of these tasks differences. Instead, it will be necessary to

579 design fMRI protocols that probe the within-subject variation in language lateralisation

580 across a range of tasks, while controlling for non-linguistic confounds. Conversely, the

581 strong, reliable and robust LI values provided by CTA would be more useful in cases

582 where a clear categorical decision on an individual's language lateralisation is required.

\section{Summary and conclusions}

584 This review has highlighted the high level of variation and inconsistency in the strength

585 and reliability of laterality measured using different language tasks. As per our

586 hypotheses, some of this variability in laterality is related to parameters such as the

587 region of interest and baseline task, which can have task-specific effects. In general

588 however, the current state of the literature is such that it is difficult to draw clear

589 conclusions that can be used to guide task selection. This review highlights the need for

590 more research that systematically compares laterality across different tasks in within-

591 subject designs, with rigorous matching of non-linguistic aspects of task design. 
The current review of the literature does suggest however some practical

593 recommendations that can be used to guide task design. Extensive use of verbal

594 fluency is clearly warranted given the robustness of its lateralisation; however, the

595 common employment of passive baselines should be replaced with active baselines

596 carefully chosen so as to isolate the language process of interest whilst controlling for

597 all other processes. Comparison of word generation with sentence generation offers the

598 opportunity to test predictions of models that assume stronger laterality for processing

599 of connected sentences over single words (Peelle, 2012; Poeppel, 2014). Future

600 studies should try to more closely match task demands of word generation and

601 sentence generation tasks, in order to systematically compare their strength of laterality

602 within subjects. For example, one could use the same stimuli for each task such as

603 names of different categories, with a cue indicating whether the subject should generate

604 instances of these categories (semantic fluency) or generate a sentence taking an

605 instance of this category as its head noun.

606 Evidence on semantic decision paradigms suggests that stronger laterality can be

607 observed when tasks require integration across the semantic content of different

608 concepts (e.g. semantic relatedness decision), rather than simple category membership

609 decision on single words. Similarly, where naming tasks are used, evidence suggests

610 that naming from description yields more robust laterality measurement than naming

611 from pictures; that is, an additional sentence comprehension component appears to

612 improve the lateralising power of this task. Sentence comprehension tasks themselves

613 appear to yield strong laterality; however, more work is needed to develop such tasks in

614 order to attempt to disentangle semantic and syntactic components. Indeed, this review 
615 highlights a distinct lack of tasks in language laterality research that aim to primarily

616 engage syntactic processing, reflected in the lack of consensus over the strength of its

617 hemispheric specialisation. Further work is needed to design and validate tasks that

618 isolate syntactic processing for laterality measurement. One possibility might be offered

619 by tasks involving judgements on 'jabberwocky' sentences (e.g. Fedorenko, Nieto-

620 Castanon, \& Kanwisher, 2012) in which content words are replaced by non-words (thus

621 preserving syntactic structure but removing semantic content).

622 More work is needed to investigate the potential significance of variability in

623 laterality across different language functions, both within individuals and at a group

624 level. Growing appreciation of the potential significance of cases of dissociated

625 dominance, both in clinical and healthy samples, should encourage the field to move

626 away from the use of single tasks and single ROIs. Instead, research should focus on

627 developing batteries of closely matched tasks that tap a variety of language functions to

628 allow systematic comparisons in within-subject studies. This will ultimately allow for

629 more quantitative meta-analyses of such literature, to draw stronger conclusions as to

630 patterns of laterality across different components of the language network.

631 One way to approach this would be to develop a generic task format in which the

632 participant is always performing the same form of task with the same type of stimuli but

633 with regards to different linguistic parameters. For example, one such format could be a

634 decision task in which one must decide if pairs of word stimuli are 'matching' or 'non-

635 matching'. The parameters that define matching and non-matching pairs can then be

636 varied according to the language process of interest; for example, rhyming versus non-

637 rhyming (phonology), same semantic category or different semantic category 
638 (semantics) or same syntactic category or different syntactic category (syntax). These 639 could be interleaved, with a visual cue indicating which decision should be made on the 640 current trial. In this way, a generic task format would remove non-linguistic differences 641 in task design that can confound interpretation of differences in laterality. This type of 642 approach has been embraced by Price and colleagues in the development of a battery 643 of tasks devised for a within-subject, fully balanced factorial design, with tasks 644 corresponding to all possible combinations of levels of factors relating to experimental 645 design aspects (e.g. stimulus modality, linguistic content, form of response). This has been used to test contrasts that allow fractionation of different levels of linguistic processing for localization of brain activity (e.g. Hope et al., 2014); future work could implement a similar battery of balanced tasks for lateralisation measurement.

CTA can provide an efficient method of isolating language activity shared across multiple different aspects of language functioning, to allow robust and reliable measurement of laterality of the core language network. However, this methodology appears to be motivated by an implicit assumption pervasive across much laterality research that there is a single core language network which displays a unitary and perfect lateralisation; thus the ability of an fMRI protocol to provide a good measure of language laterality depends on its ability to uniquely engage this language network and to yield a laterality index of close to 1 at the group level. Tasks which yield LIs further from 1 therefore are viewed as inadequate measures of language lateralisation.

We argue that defining the sensitivity of a task to capture the 'true' lateralisation 659 of a language function in terms of the strength of its laterality can be challenged. Such 660 an approach would lead one to reject tasks that yield lower LIs, which may in fact reflect 
661 meaningful variation in hemispheric organisation within the language network. For

662 example, naming is a complex linguistic function that requires both receptive and

663 expressive components, with access to both semantics and phonology; however the

664 evidence reviewed here shows it yields low LI values (e.g. Deblaere et al., 2002). Is it

665 appropriate to conclude that naming is therefore a 'poor' measure of language network

666 function? Or rather, could this tell us something about the hemispheric organisation of

667 the language functions on which it relies? We would argue that research should be

668 open to the possibility that it may be possible to validly and reliably measure laterality

669 for a language process, and yet still obtain a low LI.

670

This raises the question of how one should judge laterality paradigms; what

671 metric should one use for judging 'success' in accurately measuring an individual's

672 laterality? This review has highlighted how different methods of laterality measurement

673 can result in variable LI values for an individual across different regions, active language

674 tasks and baseline tasks. For example, in the case of verbal fluency tasks, an individual

675 may show a stronger LI when an active rather than a passive baseline is used (Dodoo-

676 Schittko et al., 2012). How should one then decide which of these can be considered to

677 best reflect the 'true' laterality of an individual? In this case, the greater strength of

678 laterality with an active baseline is often taken to indicate that this is a more accurate

679 laterality measurement; however, other metrics such as the reliability of the laterality

680 and in clinical work its predictiveness of post-surgical outcomes may arguably represent

681 better standards for assessing goodness of laterality measurement. In this way, it will be

682 important for the field to consider more deeply the metrics that are used to compare the 
683

684

685

686

687

688

689

690

691

692

693

694

695

696

697

698

699

700

701

702

703

704

705

706

707

708

709

710

711

712

713

714

715

716

717

718

719

relative utility of LIs yielded by different paradigms, and to challenge the implicit

'strongest is best' assumption that commonly guides interpretation of task LI values.

\section{References}

Baciu, M. V, Watson, J. M., Maccotta, L., McDermott, K. B., Buckner, R. L., Gilliam, F. G., \& Ojemann, J. G. (2005). Evaluating functional MRI procedures for assessing hemispheric language dominance in neurosurgical patients. Neuroradiology, 47(11), 835-844. https://doi.org/10.1007/s00234-005-1431-3

Backes, W. H., Deblaere, K., Vonck, K., Kessels, A. G., Boon, P., Hofman, P., ... Aldenkamp, A. P. (2005). Language activation distributions revealed by fMRI in post-operative epilepsy patients: Differences between left- and right-sided resections. Epilepsy Research, 66(1-3), 1-12. https://doi.org/10.1016/j.eplepsyres.2005.06.007

Bethmann, A., Tempelmann, C., Bleser, R. De, Scheich, H., \& Brechmann, A. (2007). Determining language laterality by $\mathrm{fMRI}$ and dichotic listening. Brain Research, 1133(1), 145-157. https://doi.org/10.1016/j.brainres.2006.11.057

Binder, J. R., Swanson, S. J., Hammeke, T. A., \& Sabsevitz, D. S. (2008). A comparison of five fMRI protocols for mapping speech comprehension systems. Epilepsia, 49(12), 1980-1997. https://doi.org/10.1111/j.1528-1167.2008.01683.x

Bishop, D. V. M. (2013). Cerebral Asymmetry and Language Development: Cause, Correlate, or Consequence? Science, 340(6138), 1230531. https://doi.org/10.1126/science.1230531

Bradshaw, A. R., Bishop, D. V. M., \& Woodhead, Z. V. J. (2017). Methodological considerations in assessment of language lateralisation with $\mathrm{fMRI}$ : A systematic review. PeerJ, 2017(7). https://doi.org/10.7717/peerj.3557

Brennan, N. M. P., Whalen, S., de Morales Branco, D., O'Shea, J. P., Norton, I. H., \& Golby, A. J. (2007). Object naming is a more sensitive measure of speech localization than number counting: Converging evidence from direct cortical stimulation and fMRI. Neurolmage, 37, S100-S108. https://doi.org/10.1016/j.neuroimage.2007.04.052

Clements, A. M., Rimrodt, S. L., Abel, J. R., Blankner, J. G., Mostofsky, S. H., Pekar, J. J., ... Cutting, L. E. (2006). Sex differences in cerebral laterality of language and visuospatial processing. Brain and Language, 98(2), 150-158. https://doi.org/https://doi.org/10.1016/j.bandl.2006.04.007

Cousin, E., Peyrin, C., Pichat, C., Lamalle, L., Bas, J.-F. Le, \& Baciu, M. (2007). Functional MRI approach for assessing hemispheric predominance of regions activated by a phonological and a semantic task. European Journal of Radiology, 63(2), 274-285. https://doi.org/10.1016/j.ejrad.2007.01.030 
720

721

722

723

724

725

726

727

728

729

730

731

732

733

734

735

736

737

738

739

740

741

742

743

744

745

746

747

748

749

750

751

752

753

754

755

756

757

758

759

Deblaere, K., Backes, W. H., Hofman, P., Vandemaele, P., Boon, P. A., Vonck, K., ... Aldenkamp, A. (2002). Developing a comprehensive presurgical functional MRI protocol for patients with intractable temporal lobe epilepsy: a pilot study. Neuroradiology, 44(8), 667-673. https://doi.org/10.1007/s00234-002-0800-4

der Haegen, L. Van, Cai, Q., \& Brysbaert, M. (2012). Colateralization of Broca's area and the visual word form area in left-handers: fMRI evidence. Brain and Language, 122(3), 171-178. https://doi.org/10.1016/j.bandl.2011.11.004

Dodoo-Schittko, F., Rosengarth, K., Doenitz, C., \& Greenlee, M. W. (2012). Assessing language dominance with functional MRI: The role of control tasks and statistical analysis. Neuropsychologia, 50(11), 2684-2691. https://doi.org/10.1016/j.neuropsychologia.2012.07.032

Fedorenko, E., Nieto-Castanon, A., \& Kanwisher, N. (2012). Lexical and syntactic representations in the brain: An fMRI investigation with multi-voxel pattern analyses. Neuropsychologia, 50(4), 499-513. https://doi.org/10.1016/j.neuropsychologia.2011.09.014

Fernandez, G., de Greiff, A., von Oertzen, J., Reuber, M., Lun, S., Klaver, P., ... Elger, C. E. (2001). Language mapping in less than 15 minutes: Real-time functional MRI during routine clinical investigation. Neurolmage, 14(3), 585-594. https://doi.org/10.1006/nimg.2001.0854

Friederici, A. D. (2011). The Brain Basis of Language Processing: from Structure to Function. Physiological Reviews, 91(4), 1357-1392. https://doi.org/10.1152/physrev.00006.2011

Gaillard, W. D., Balsamo, L., Xu, B., Grandin, C. B., Braniecki, S. H., Papero, P. H., ... Theodore, W. H. (2002). Language dominance in partial epilepsy patients identified with an fMRI reading task. Neurology, 59(2), 256-265.

Gaillard, W. D., Sachs, B. C., Whitnah, J. R., Ahmad, Z., Balsamo, L. M., Petrella, J. R., ... Grandin, C. B. (2003). Developmental aspects of language processing: fMRI of verbal fluency in children and adults. Human Brain Mapping, 18(3), 176-185. https://doi.org/10.1002/hbm.10091

Häberling, I. S., Steinemann, A., \& Corballis, M. C. (2016). Cerebral asymmetry for language: Comparing production with comprehension. Neuropsychologia, 80, 17-23. https://doi.org/10.1016/j.neuropsychologia.2015.11.002

Harrington, G. S., Buonocore, M. H., \& Farias, S. T. (2006). Intrasubject reproducibility of functional MR imaging activation in language tasks. American Journal of Neuroradiology, 27(4), 938-944.

Harris, P. A., Taylor, R., Thielke, R., Payne, J., Gonzalez, N., \& Conde, J. G. (2009). Research electronic data capture (REDCap)-A metadata-driven methodology and workflow process for providing translational research informatics support. Journal of Biomedical Informatics, 42(2), 377-381. https://doi.org/10.1016/j.jbi.2008.08.010 
760

761

762

763

764

765

766

767

768

769

770

771

772

773

774

775

776

777

778

779

780

781

782

783

784

785

786

787

788

789

790

791

792

793

794

795

796

797

798

Hickok, G., \& Poeppel, D. (2007). The cortical organization of speech processing. Nature Reviews Neuroscience, 8(5), 393-402. https://doi.org/10.1038/nrn2113

Hillis, A. E. (2007). Aphasia - Progress in the last quarter of a century. Neurology, 69(2), 200-213. https://doi.org/10.1212/01.wnl.0000265600.69385.6f

Hope, T. M. H., Prejawa, S., Parker Jones, 'Ōiwi, Oberhuber, M., Seghier, M. L., Green, D. W., \& Price, C. J. (2014). Dissecting the functional anatomy of auditory word repetition. Frontiers in Human Neuroscience, 8, 246. https://doi.org/10.3389/fnhum.2014.00246

Hund-Georgiadis, M., Lex, U., Friederici, A. D., \& von Cramon, D. Y. (2002). Noninvasive regime for language lateralization in right- and left-handers by means of functional MRI and dichotic listening. Experimental Brain Research, 145(2), 166176. https://doi.org/10.1007/s00221-002-1090-0

Hund-Georgiadis, M., Lex, U., \& von Cramon, D. Y. (2001). Language dominance assessment by means of fMRI: Contributions from task design, performance, and stimulus modality. Journal of Magnetic Resonance Imaging, 13(5), 668-675. https://doi.org/10.1002/jmri.1094

Jansen, A., Menke, R., Sommer, J., Förster, A. F., Bruchmann, S., Hempleman, J., ... Knecht, S. (2006). The assessment of hemispheric lateralization in functional MRI-Robustness and reproducibility. Neurolmage, 33(1), 204-217. https://doi.org/10.1016/j.neuroimage.2006.06.019

Jensen-Kondering, U. R., Ghobadi, Z., Wolff, S., Jansen, O., \& Ulmer, S. (2012). Acoustically presented semantic decision-making tasks provide a robust depiction of the temporo-parietal speech areas. Journal of Clinical Neuroscience, 19(3), 428-433. https://doi.org/10.1016/j.jocn.2011.04.038

Kennan, R. P., Kim, D., Maki, A., Koizumi, H., \& Constable, R. T. (2002). Non-invasive assessment of language lateralization by transcranial near infrared optical topography and functional MRI. Human Brain Mapping, 16(3), 183-189. Retrieved from http://ovidsp.ovid.com/ovidweb.cgi?T=JS\&CSC=Y\&NEWS=N\&PAGE=fulltext\&D= psyc4\&AN=2002-17307-005;

Kleinhans, N. M., Mueller, R.-A., Cohen, D. N., \& Courchesne, E. (2008). Atypical functional lateralization of language in autism spectrum disorders. Brain Research, 1221, 115-125. https://doi.org/10.1016/j.brainres.2008.04.080

Liberman, A. M., \& Mattingly, I. G. (1985). The Motor Theory of Speech-Perception Revised. Cognition, 21(1), 1-36. https://doi.org/10.1016/0010-0277(85)90021-6

Mazoyer, B., Zago, L., Jobard, G., Crivello, F., Joliot, M., Perchey, G., ... TzourioMazoyer, N. (2014). Gaussian mixture modeling of hemispheric lateralization for language in a large sample of healthy individuals balanced for handedness. PLoS ONE. https://doi.org/10.1371/journal.pone.0101165 
799

800

801

802

803

804

805

806

807

808

809

810

811

812

813

814

815

816

817

818

819

820

821

822

823

824

825

826

827

828

829

830

831

832

833

834

835

836

837

838

Miro, J., Ripolles, P., Lopez-Barroso, D., Vila-Ballo, A., Juncadella, M., de DiegoBalaguer, R., ... Falip, M. (2014). Atypical language organization in temporal lobe epilepsy revealed by a passive semantic paradigm. Bmc Neurology, 14, 98. https://doi.org/10.1186/1471-2377-14-98

Moher, D., Liberati, A., Tetzlaff, J., \& Altman, D. G. (2009). Preferred reporting items for systematic reviews and meta-analyses: the PRISMA statement. BMJ, 339. Retrieved from http://www.bmj.com/content/339/bmj.b2535.abstract

Morrison, M. A., Churchill, N. W., Cusimano, M. D., Schweizer, T. A., Das, S., \& Graham, S. J. (2016). Reliability of task-based fMRI for preoperative planning: A test-retest study in brain tumor patients and healthy controls. PLoS ONE. https://doi.org/10.1371/journal.pone.0149547

Niskanen, E., Kononen, M., Villberg, V., Nissi, M., Ranta-aho, P., Saisanen, L., ... Vanninen, R. (2012). The effect of fMRI task combinations on determining the hemispheric dominance of language functions. Neuroradiology, 54(4), 393-405. https://doi.org/10.1007/s00234-011-0959-7

Ocklenburg, S., Hugdahl, K., \& Westerhausen, R. (2013). Structural white matter asymmetries in relation to functional asymmetries during speech perception and production. Neurolmage, 83, 1088-1097. https://doi.org/https://doi.org/10.1016/j.neuroimage.2013.07.076

Partovi, S., Jacobi, B., Rapps, N., Zipp, L., Karimi, S., Rengier, F., ... Stippich, C. (2012). Clinical Standardized fMRI Reveals Altered Language Lateralization in Patients with Brain Tumor. American Journal of Neuroradiology, 33(11), 21512157. https://doi.org/10.3174/ajnr.A3137

Partovi, S., Konrad, F., Karimi, S., Rengier, F., Lyo, J. K., Zipp, L., ... Stippich, C. (2012). Effects of Covert and Overt Paradigms in Clinical Language fMRI. Academic Radiology, 19(5), 518-525. https://doi.org/10.1016/j.acra.2011.12.017

Patterson, K., Nestor, P. J., \& Rogers, T. T. (2007). Where do you know what you know? The representation of semantic knowledge in the human brain. Nat Rev Neurosci, 8(12), 976-987. Retrieved from http://dx.doi.org/10.1038/nrn2277

Peelle, J. (2012). The hemispheric lateralization of speech processing depends on what "speech" is: a hierarchical perspective. Frontiers in Human Neuroscience, 6, 309. https://doi.org/10.3389/fnhum.2012.00309

Poeppel, D. (2014). The neuroanatomic and neurophysiological infrastructure for speech and language. Current Opinion in Neurobiology, 28, 142-149. https://doi.org/10.1016/j.conb.2014.07.005

Price, C. J. (2012). A review and synthesis of the first 20 years of PET and fMRI studies of heard speech, spoken language and reading. Neurolmage, 62(2), 816-847. https://doi.org/10.1016/j.neuroimage.2012.04.062

Propper, R. E., O'Donnell, L. J., Whalen, S., Tie, Y., Norton, I. H., Suarez, R. O., ... Golby, A. J. (2010). A combined fMRI and DTI examination of functional 
language lateralization and arcuate fasciculus structure: Effects of degree versus direction of hand preference. Brain and Cognition, 73(2), 85-92. https://doi.org/https://doi.org/10.1016/j.bandc.2010.03.004

Ramsey, N. F., Sommer, I. E. C., Rutten, G. J., \& Kahn, R. S. (2001). Combined analysis of language tasks in fMRI improves assessment of hemispheric dominance for language functions in individual subjects. Neurolmage, 13(4), 719-733. https://doi.org/10.1006/nimg.2000.0722

Rasmussen, T., \& Milner, B. (1975). Clinical and surgical studies of the cerebral speech areas in man. In K. J. Zulch, O. Creutzfeldt, \& G. C. Galbraith (Eds.) (p. 238). Berlin: Springer.

Razafimandimby, A., Maiza, O., Herve, P.-Y., Lecardeur, L., Delamillieure, P., Brazo, P., ... Dollfus, S. (2007). Stability of functional language lateralization over time in schizophrenia patients. Schizophrenia Research, 94(1-3), 197-206. https://doi.org/10.1016/j.schres.2007.04.011

Rice, G. E., Ralph, M. A. L., \& Hoffman, P. (2015). The Roles of Left Versus Right Anterior Temporal Lobes in Conceptual Knowledge: An ALE Meta-analysis of 97 Functional Neuroimaging Studies. Cerebral Cortex, 25(11), 4374-4391. https://doi.org/10.1093/cercor/bhv024

Ruff, I. M., Brennan, N. M. P., Peck, K. K., Hou, B. L., Tabar, V., Brennan, C. W., \& Holodny, A. I. (2008). Assessment of the language laterality index in patients with brain tumor using functional MR imaging: Effects of thresholding, task selection, and prior surgery. American Journal of Neuroradiology, 29(3), 528-535. https://doi.org/10.3174/ajnr.A0841

Rutten, G. J. M., Ramsey, N. F., van Rijen, P. C., \& van Veelen, C. W. M. (2002). Reproducibility of $\mathrm{fMRI}$-determined language lateralization in individual subjects. Brain and Language, 80(3), 421-437. https://doi.org/10.1006/brln.2001.2600

Sanjuan, A., Bustamante, J.-C., Forn, C., Ventura-Campos, N., Barros-Loscertales, A., Martinez, J.-C., ... Avila, C. (2010). Comparison of two fMRI tasks for the evaluation of the expressive language function. Neuroradiology, 52(5), 407-415. https://doi.org/10.1007/s00234-010-0667-8

Sanjuan, A., Forn, C., Ventura-Campos, N., Rodriguez-Pujadas, A., Garcia-Porcar, M., Belloch, V., ... Avila, C. (2010). The sentence verification task: a reliable fMRI protocol for mapping receptive language in individual subjects. European Radiology, 20(10), 2432-2438. https://doi.org/10.1007/s00330-010-1814-7

Seghier, M. L., Lazeyras, F., Pegna, A. J., Annoni, J.-M., Zimine, I., Mayer, E., ... Khateb, A. (2004). Variability of fMRI activation during a phonological and semantic language task in healthy subjects. Human Brain Mapping, 23(3), 140155. https://doi.org/10.1002/hbm.20053

Sommer, I. E. C., Ramsey, N. F., Mandl, R. C. W., \& Kahn, R. S. (2003). Language lateralization in female patients with schizophrenia: an fMRI study. Schizophrenia Research, 60(2-3), 183-190. https://doi.org/10.1016/S0920-9964(02)00300-6 
880

881

882

883

884

885

886

887

888

889

890

891

892

893

894

895

896

897

898

899

900

901

902

903

904

905

906

907

908

909

910

911

912

913

914

915

916

917

918

919

920

Stippich, C., Mohammed, J., Kress, B., Hahnel, S., Gunther, J., Konrad, F., \& Sartor, K. (2003). Robust localization and lateralization of human language function: an optimized clinical functional magnetic resonance imaging protocol. Neuroscience Letters, 346(1-2), 109-113. https://doi.org/10.1016/S0304-3940(03)00561-5

Szaflarski, J. P., Holland, S. K., Jacola, L. M., Lindsell, C., Privitera, M. D., \& Szaflarski, M. (2008). Comprehensive presurgical functional MRI language evaluation in adult patients with epilepsy. Epilepsy \& Behavior, 12(1), 74-83. https://doi.org/https://doi.org/10.1016/j.yebeh.2007.07.015

Tailby, C., Weintrob, D. L., Saling, M. M., Fitzgerald, C., \& Jackson, G. D. (2014). Reading difficulty is associated with failure to lateralize temporooccipital function. Epilepsia, 55(5), 746-753. https://doi.org/10.1111/epi.12607

Thivard, L., Hombrouck, J., du Montcel, S. T., Delmaire, C., Cohen, L., Samson, S., ... Lehericy, S. (2005). Productive and perceptive language reorganization in temporal lobe epilepsy. Neurolmage, 24(3), 841-851. https://doi.org/10.1016/j.neuroimage.2004.10.001

Tyler, L. K., Marslen-Wilson, W. D., Randall, B., Wright, P., Devereux, B. J., Zhuang, J., ... Stamatakis, E. A. (2011). Left inferior frontal cortex and syntax: function, structure and behaviour in patients with left hemisphere damage. Brain, 134, 415-431. https://doi.org/10.1093/brain/awq369

Tzourio-Mazoyer, N., Joliot, M., Marie, D., \& Mazoyer, B. (2016). Variation in homotopic areas' activity and inter-hemispheric intrinsic connectivity with type of language lateralization: an FMRI study of covert sentence generation in 297 healthy volunteers. Brain Structure \& Function, 221(5), 2735-2753. https://doi.org/10.1007/s00429-015-1068-x

Tzourio-Mazoyer, N., Marie, D., Zago, L., Jobard, G., Perchey, G., Leroux, G., ... Mazoyer, B. (2015). Heschl's gyrification pattern is related to speech-listening hemispheric lateralization: FMRI investigation in 281 healthy volunteers. Brain Structure \& Function, 220(3), 1585-1599. https://doi.org/10.1007/s00429-0140746-4

van Oers, C. A. M. M., Vink, M., van Zandvoort, M. J. E., van der Worp, H. B., de Haan, E. H. F., Kappelle, L. J., ... Dijkhuizen, R. M. (2010). Contribution of the left and right inferior frontal gyrus in recovery from aphasia. A functional MRI study in stroke patients with preserved hemodynamic responsiveness. Neurolmage, 49(1), 885-893. https://doi.org/10.1016/j.neuroimage.2009.08.057

van Rijn, S., Aleman, A., Swaab, H., Vink, M., Sommer, I., \& Kahn, R. S. (2008). Effects of an extra $X$ chromosome on language lateralization: An fMRI study with Klinefelter men (47,XXY). Schizophrenia Research, 101(1-3), 17-25. https://doi.org/10.1016/j.schres.2008.02.001

Vassal, F., Schneider, F., Boutet, C., Jean, B., Sontheimer, A., \& Lemaire, J.-J. (2016). Combined DTI Tractography and Functional MRI Study of the Language Connectome in Healthy Volunteers: Extensive Mapping of White Matter Fascicles 
and Cortical Activations. Plos One, 11(3), e0152614.

https://doi.org/10.1371/journal.pone.0152614

Vernooij, M. W., Smits, M., Wielopolski, P. A., Houston, G. C., Krestin, G. P., \& van der Lugt, A. (2007). Fiber density asymmetry of the arcuate fasciculus in relation to functional hemispheric language lateralization in both right- and left-handed healthy subjects: A combined fMRI and DTI study. Neurolmage, 35(3), 10641076. https://doi.org/https://doi.org/10.1016/j.neuroimage.2006.12.041

Vikingstad, E. M., George, K. P., Johnson, A. F., \& Cao, Y. (2000). Cortical language lateralization in right handed normal subjects using functional magnetic resonance imaging. Journal of the Neurological Sciences, 175(1), 17-27. https://doi.org/10.1016/S0022-510X(00)00269-0

Vingerhoets, G., Alderweireldt, A.-S., Vandemaele, P., Cai, Q., der Haegen, L. Van, Brysbaert, M., \& Achten, E. (2013). Praxis and language are linked: Evidence from co-lateralization in individuals, with atypical language dominance. Cortex, 49(1), 172-183. https://doi.org/10.1016/j.cortex.2011.11.003

Warrington, E. K., \& McCarthy, R. A. (1987). Categories of Knowledge - further Fractionations and an Attempted Integration. Brain, 110, 1273-1296. https://doi.org/10.1093/brain/110.5.1273

Warrington E. K., \& McCarthy, R. A (1983). Category specific access dysphasia. Brain, 106(4), 859. https://doi.org/https://ezproxyprd.bodleian.ox.ac.uk:4563/10.1093/brain/106.4.859

Warrington, E. K., \& Shallice, T. (1984). Category Specific Semantic Impairments. Brain, 107(SEP), 829-854. https://doi.org/10.1093/brain/107.3.829

Wright, P., Stamatakis, E. A., \& Tyler, L. K. (2012). Differentiating Hemispheric Contributions to Syntax and Semantics in Patients with Left-Hemisphere Lesions. Journal of Neuroscience, 32(24), 8149-8157. https://doi.org/10.1523/JNEUROSCI.0485-12.2012

Zaca, D., Jarso, S., \& Pillai, J. J. (2013). Role of Semantic Paradigms for Optimization of Language Mapping in Clinical fMRI Studies. American Journal of Neuroradiology, 34(10), 1966-1971. https://doi.org/10.3174/ajnr.A3628 
Figure 1

\section{Literature search and selection process}

Flow diagram illustrating the search and selection process for obtaining papers for inclusion in the review. Adapted from Moher, Liberati, Tetzlaff and Altman (2009).
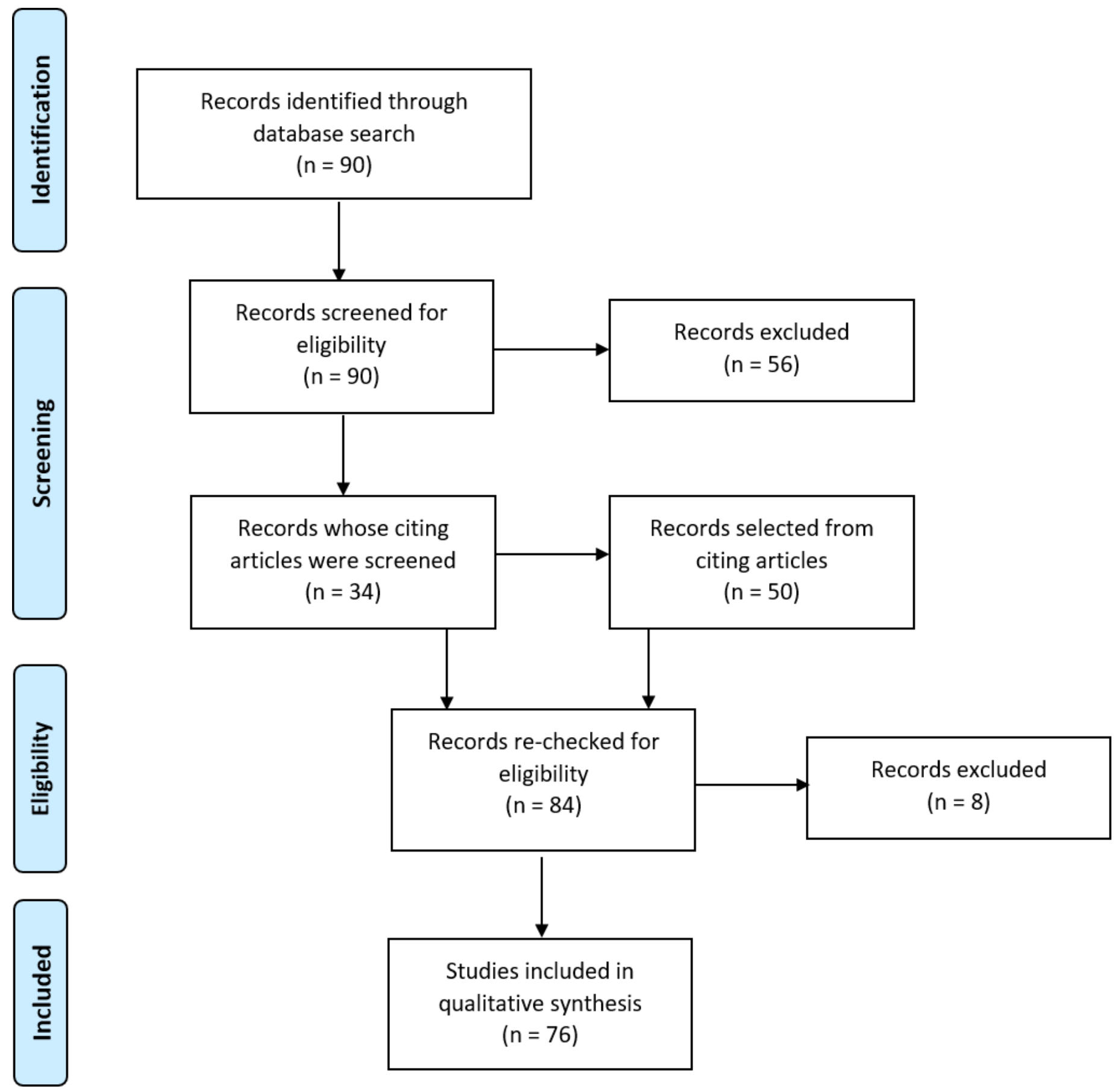


\section{Figure 2}

\section{Forest plot of mean LI values for verbal fluency}

Forest plot shows mean LI values for verbal fluency tasks reported from studies meeting our criteria. Plot is divided up according to region of interest used for $\mathrm{LI}$ calculation (frontal, temporoparietal, combined frontal and temporoparietal and global). Error bars represent $95 \%$ confidence limits. Colour of symbol indicates type of baseline task used (active or passive), and shape of symbol indicates method of LI calculation (see key). *Papers did not report a measure of spread for $\mathrm{LI}$ values, so confidence interval is not shown. ** $\mathrm{LI}$ values reported by this paper are given at different thresholds: $Z=5.3$ (Top), $Z=2.3$ (Bottom). Figures 2,3 and 4 are published on Figshare and can be found at:

https://figshare.com/articles/Forrest_Plots_of_LI_values_for_different_language tasks/4977950

\section{Method}
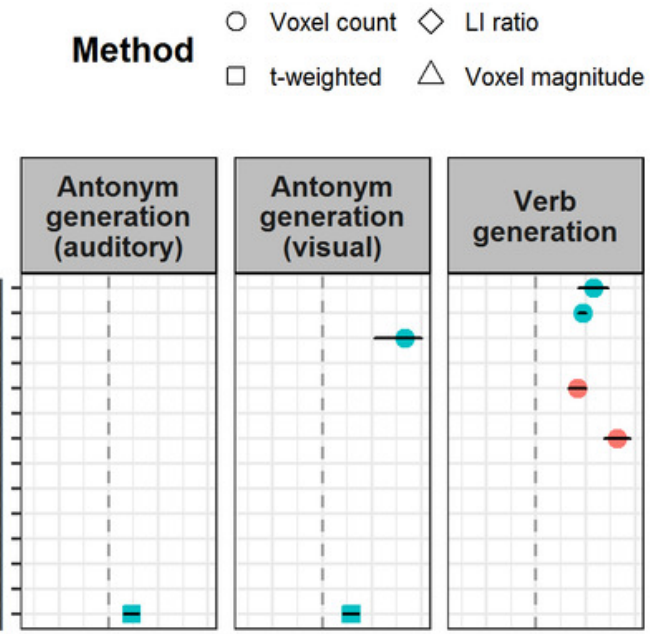

Vikingstad et al (2000), $\mathrm{N}=23]$ van Oers et al 2010$),[\mathrm{N}=13$
Tie et al $(2009),[\mathrm{N}=6$ Thivard et al (2005), $\mathrm{N}=17$ Szaflarski et al (2008), $\mathrm{N}=49$

Stippich et al 2003$\},, N=14$.

Sanjuan et al (2010), $\mathrm{N}=18$

Partovi et al (2012b),, $\mathrm{N}=14$

Partovi et al 2012a),, $\mathrm{N}=14$ Ocklenburg et al (2013),, $\mathrm{N}=29$

Gaillard et al (2003), 'N=22

Adcock et al 2003,', $\mathrm{N}=12$

*Sanjuan et al 2010), $\mathrm{N}=18$

Suarez et al (2007), [ $\mathrm{N}=13$.

van Oers et al (2010), $[\mathrm{N}=13]$

Thivard et al 2005 , ,, $\mathrm{N}=17$

Szaflarski et al 2008), $\mathrm{N}=49$

Stippich et al (2003), $\mathrm{N}=14$

Partovi et al (2012b), $\mathrm{N}=14$

Partovi et al 2012a),, $\mathrm{N}=14$

Ocklenburg et al (2013),, $\mathrm{N}=29$

Gaillard et al 2003,', $\mathrm{N}=22$

*Vikingstad et al 2000, , $\mathrm{N}=23$ Tie et al (2009), $[\mathrm{N}=6$.

Suarez et al (2007), $[\mathrm{N}=13]$

van Oers et al (2010), $\mathrm{N}=13]$ Tailby et al (2014),, $\mathrm{N}=42$

Szaflarski et al 2008), $\mathrm{N}=49$.

Sveller et al 2006), $\mathrm{N}=63$

Pravata et al 2011), $\mathrm{N}=12$

Dodoo-Schittko et al (2012), $\mathrm{N}=11$

Backes et al (2005), [N=9

Baciu et al (2005),, $\mathrm{N}=10$

**Adcock et al (2003),, $\mathrm{N}=12$

Suarez et al (2007), $[\mathrm{N}=13]$

Pravata et al 2011), $(\mathrm{N}=12$

Krinik et al (2003), $[\mathrm{N}=6$.

*Deblaere et al (2002), [ $\mathrm{N}=9$ ] -
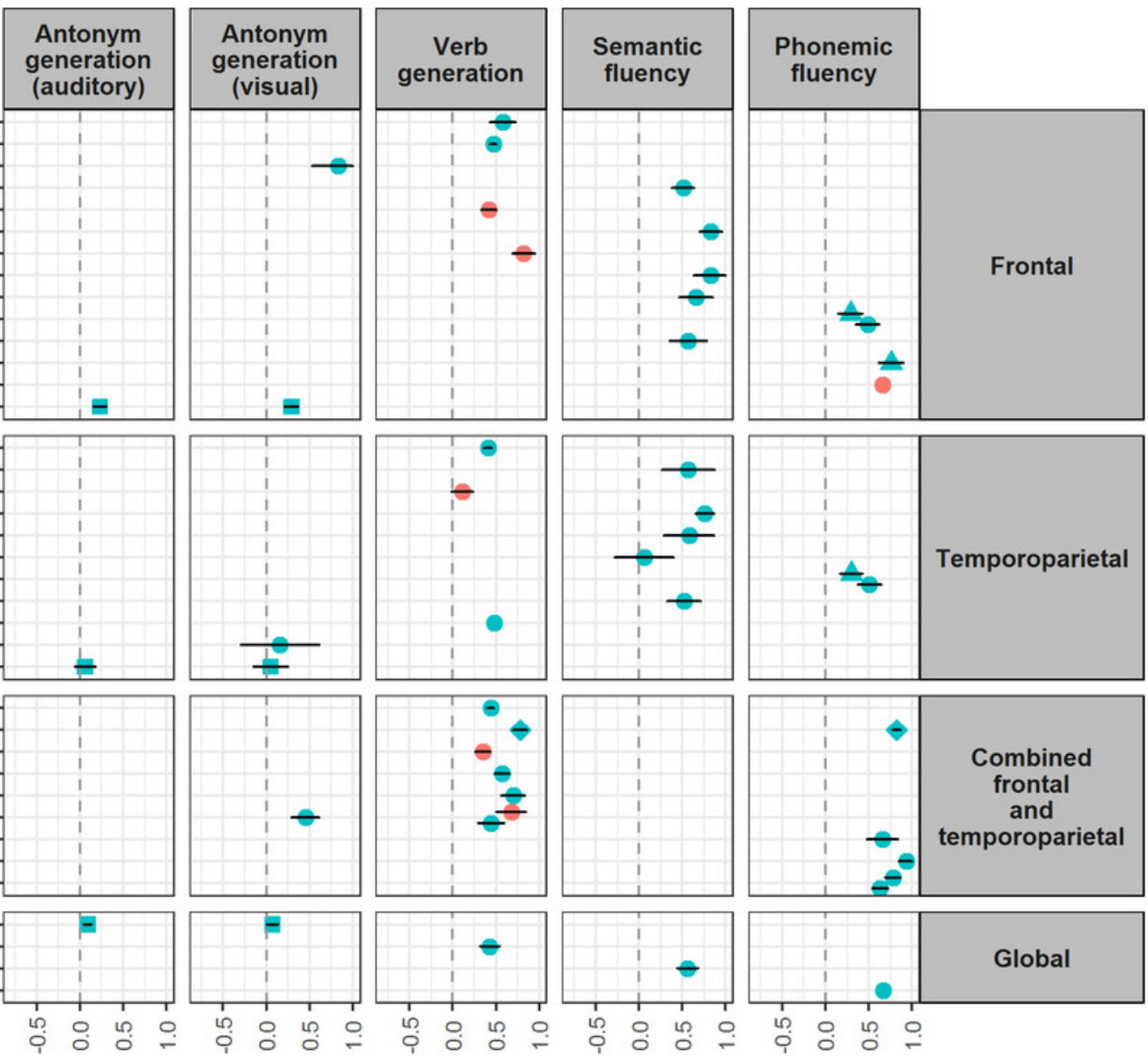

LI 


\section{Figure 3}

\section{Forest plot of mean LI values for phonemic judgement, naming, sentence comprehension and sentence generation tasks.}

Forest plot shows mean LI values for different language tasks reported from studies meeting our criteria. Plot is divided up according to region of interest used for $\mathrm{LI}$ calculation (frontal, temporoparietal, combined frontal and temporoparietal and global). Error bars represent 95\% confidence limits. Colour of symbol indicates type of baseline task used (active or passive), and shape of symbol indicates method of $\mathrm{LI}$ calculation (see key). *Papers did not report a measure of spread for LI values, so confidence interval is not shown. ${ }^{* *} \mathrm{LI}$ values reported by this paper are given at different thresholds: $t=5$ (Top), $t=4$ (Middle) and $t$ $=3$ (Bottom).

Method

$\bigcirc$ Voxel count $\nabla$ Flip method

$\diamond$ LI ratio
Baseline Active
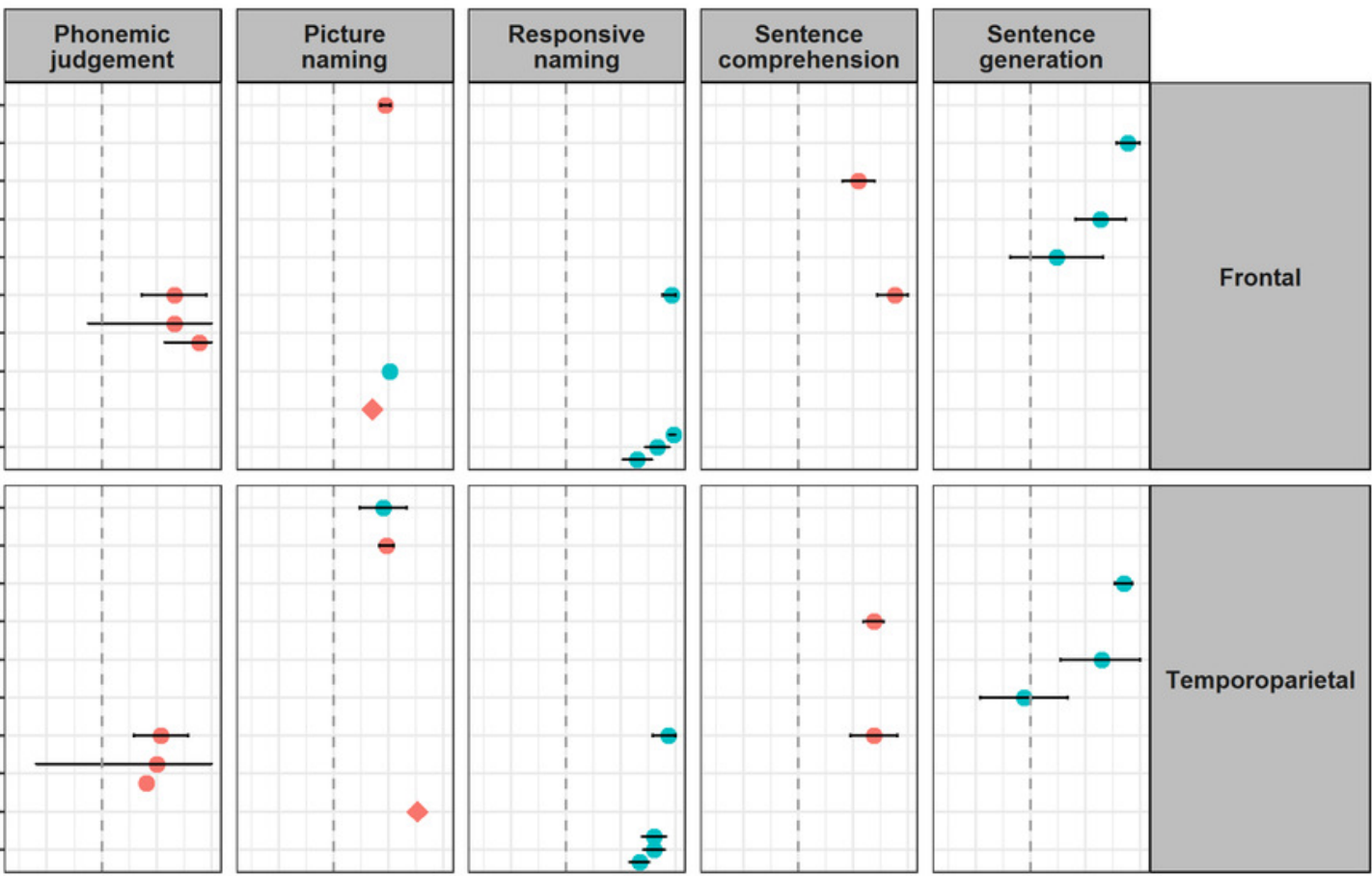

Van Oers et al (2010), [N=13] Niskanen et al (2012), [N=20] Baciu et al (2005), [N=10]

Vassal et al (2016), [N=20]Seghier et al (2004), [N=26] Kennan et al (2002), [N=6] *Deblaere et al (2002), [N=9] -
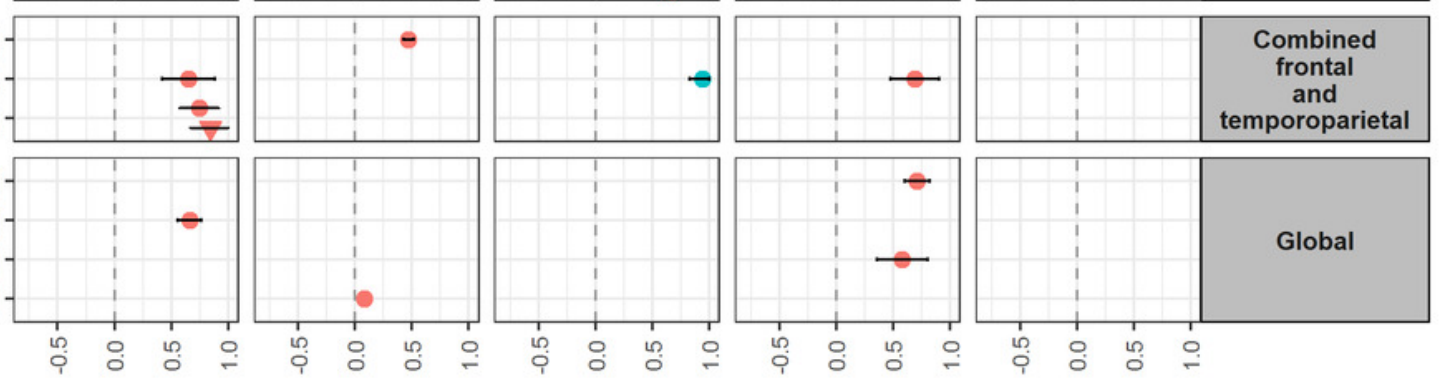

LI 


\section{Figure 4}

Forest plot of mean LI values for semantic decision, text reading and speech listening tasks.

Forest plot shows mean LI values for different language tasks reported from studies meeting our criteria. Plot is divided up according to region of interest used for LI calculation (frontal, temporoparietal, combined frontal and temporoparietal and global). Error bars represent $95 \%$ confidence limits. Colour of symbol indicates type of baseline task used (active or passive), and shape of symbol indicates method of LI calculation (see key).*Papers did not report a measure of spread for LI values, so confidence interval is not shown.

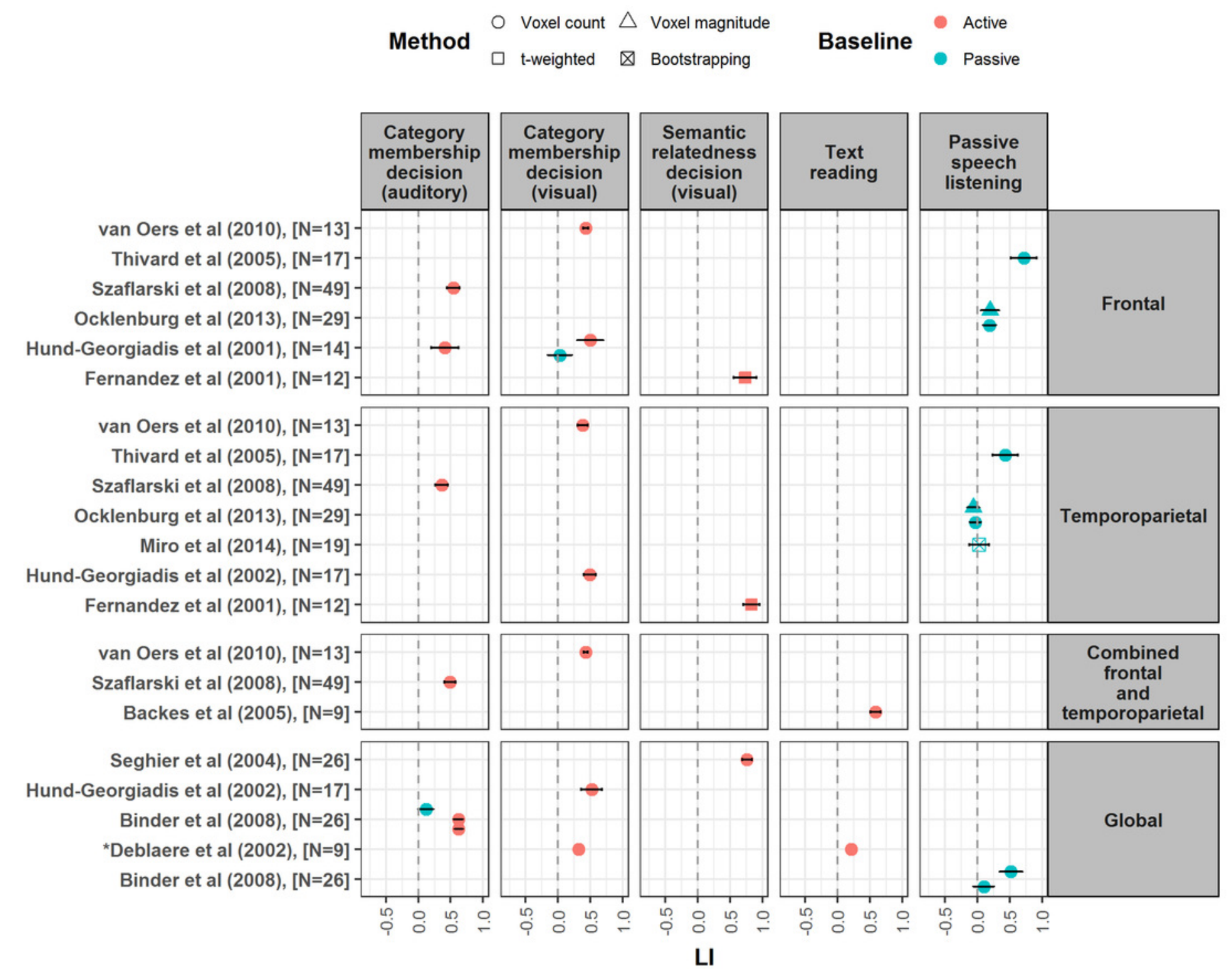




\section{Table $\mathbf{1}$ (on next page)}

Model-based predictions of language lateralisation

Table illustrates some predictions of different models of the neural basis of language, in terms of the lateralisation expected for different aspects of language processing. $\mathrm{B}=$ Bilateral, $\mathrm{L}=$ Lateralised. 


\begin{tabular}{|c|c|c|c|c|}
\hline $\begin{array}{l}\text { Theoretical } \\
\text { principle/ Model }\end{array}$ & $\begin{array}{c}\text { Speech } \\
\text { acoustic } \\
\text { processing }\end{array}$ & $\begin{array}{c}\text { Speech } \\
\text { comprehension }\end{array}$ & $\begin{array}{l}\text { Speech } \\
\text { articulation }\end{array}$ & Semantics \\
\hline
\end{tabular}

\section{Dual stream} model of speech processing

B

(Hickok \&

Poeppel., 2007)

Hierarchical asymmetry of linguistic complexity (Peelle., 2012)

Bilateral sensorimotor inputs/outputs and left lateralised central language processes (Price., 2012)

COM-PRE hypothesis (Poeppel., 2014)

B

B

B
B

L

B

L
B

$\mathrm{L}$

L 


\section{Table 2 (on next page)}

Language processes engaged by different language and baseline tasks.

Table shows the main language tasks (top left quadrant) and baseline tasks (bottom left quadrant) identified as being widely used in laterality research. For each type of task, the number of studies (N) within our search selection using this task is given, as well as one characterisation of the different language processes (middle column) and domain general processes (right column) they engage. Tick = engaged, bracketed tick = sometimes engaged (e.g. depending on task demands, modality of stimuli, occurrence of automatic linguistic processing). 


\begin{tabular}{|c|c|c|c|c|c|c|c|c|c|c|}
\hline Task & $\begin{array}{l}\mathrm{N} \\
\text { studies }\end{array}$ & $\begin{array}{l}\text { Speech } \\
\text { motor } \\
\text { planning/ } \\
\text { articulation }\end{array}$ & $\begin{array}{l}\text { Phono- } \\
\text { logical } \\
\text { access }\end{array}$ & $\begin{array}{l}\text { Ortho- } \\
\text { graphical } \\
\text { processing }\end{array}$ & Semantics & $\begin{array}{l}\text { Word } \\
\text { retrieval }\end{array}$ & Syntax & $\begin{array}{l}\text { Working } \\
\text { memory }\end{array}$ & $\begin{array}{l}\text { Motor } \\
\text { processing }\end{array}$ & $\begin{array}{l}\text { Auditory } \\
\text { processing }\end{array}$ \\
\hline Verbal fluency & 53 & $\checkmark$ & $\checkmark$ & $(\checkmark)$ & $(\checkmark)$ & $\checkmark$ & & $\checkmark$ & & \\
\hline Sentence generation & 5 & $\checkmark$ & $\checkmark$ & & $\checkmark$ & $\checkmark$ & $\checkmark$ & $\checkmark$ & & \\
\hline $\begin{array}{l}\text { Passive speech } \\
\text { listening }\end{array}$ & 7 & & $\checkmark$ & & $\checkmark$ & & $\checkmark$ & $(\checkmark)$ & & $\checkmark$ \\
\hline Text reading & 2 & $\checkmark$ & $\checkmark$ & $\checkmark$ & $\checkmark$ & & $\checkmark$ & $(\checkmark)$ & & \\
\hline Phonemic decision & 8 & $(\checkmark)$ & $\checkmark$ & $(\checkmark)$ & $(\checkmark)$ & $(\checkmark)$ & & $\checkmark$ & $(\checkmark)$ & \\
\hline Semantic decision & 20 & & $(\checkmark)$ & $(\checkmark)$ & $\checkmark$ & $(\checkmark)$ & & $\checkmark$ & $(\checkmark)$ & \\
\hline $\begin{array}{l}\text { Sentence } \\
\text { comprehension }\end{array}$ & 8 & & $\checkmark$ & $(\checkmark)$ & $\checkmark$ & & $\checkmark$ & $\checkmark$ & & $(\checkmark)$ \\
\hline Naming & 9 & $\checkmark$ & $\checkmark$ & & $\checkmark$ & $\checkmark$ & & & & \\
\hline Rest & 37 & & & & $(\checkmark)$ & & & & & \\
\hline $\begin{array}{l}\text { Perceptual decision } \\
\text { (non-linguistic) }\end{array}$ & 25 & & & & & & & $\checkmark$ & $(\checkmark)$ & \\
\hline Finger tapping & 3 & & & & & & & & $\checkmark$ & \\
\hline $\begin{array}{l}\text { Non-word/word } \\
\text { repetition }\end{array}$ & 8 & $\checkmark$ & $\checkmark$ & & & & & & & \\
\hline $\begin{array}{l}\text { Recite months of the } \\
\text { year/count sequence }\end{array}$ & 2 & $\checkmark$ & $\checkmark$ & & $(\checkmark)$ & $\checkmark$ & & & & \\
\hline Tone listening & 3 & & & & & & & & & $\checkmark$ \\
\hline $\begin{array}{l}\text { Backward speech } \\
\text { listening }\end{array}$ & 2 & & & & & & & & & $\checkmark$ \\
\hline $\begin{array}{l}\text { Nonsense text } \\
\text { reading }\end{array}$ & 2 & $\checkmark$ & $\checkmark$ & $\checkmark$ & & & & & & \\
\hline $\begin{array}{l}\text { Spatial position } \\
\text { naming }\end{array}$ & 1 & $\checkmark$ & $\checkmark$ & & $\checkmark$ & $\checkmark$ & & & & \\
\hline
\end{tabular}

$\checkmark=$ engaged

$(\checkmark)$ = sometimes engaged (e.g. depending on task demands, modality of stimuli, occurrence of automatic linguistic processing) 


\section{Table 3 (on next page)}

Language processes isolated by subtraction of different baseline tasks from sentence generation.

Table shows comparison of passive and active baseline tasks as used for subtraction with sentence generation, in terms of the language and domain-general processes engaged by each paradigm and isolated by the subtraction contrast. Tick = engaged, bracketed tick = sometimes engaged (e.g. depending on task demands, modality of stimuli, occurrence of automatic linguistic processing). 


\begin{tabular}{|c|c|c|c|c|c|c|c|c|c|}
\hline Contrast & $\begin{array}{l}\text { Speech } \\
\text { motor } \\
\text { planning/ } \\
\text { articulation }\end{array}$ & $\begin{array}{l}\text { Phono- } \\
\text { logical } \\
\text { access }\end{array}$ & $\begin{array}{l}\text { Ortho- } \\
\text { graphical } \\
\text { processing }\end{array}$ & Semantics & $\begin{array}{l}\text { Word } \\
\text { retrieval }\end{array}$ & Syntax & $\begin{array}{l}\text { Working } \\
\text { memory }\end{array}$ & $\begin{array}{l}\text { Motor } \\
\text { processing }\end{array}$ & $\begin{array}{l}\text { Auditory } \\
\text { processing }\end{array}$ \\
\hline $\begin{array}{l}\text { Task: Sentence generation } \\
\text { Baseline: Rest }\end{array}$ & $\checkmark$ & $\checkmark$ & & $\begin{array}{l}\checkmark \\
(\checkmark)\end{array}$ & $\checkmark$ & $\checkmark$ & $\checkmark$ & & \\
\hline $\begin{array}{l}\text { Sentence generation vs } \\
\text { Rest }\end{array}$ & $\checkmark$ & $\checkmark$ & & $(\checkmark)$ & $\checkmark$ & $\checkmark$ & $\checkmark$ & & \\
\hline Task: Sentence generation & $\checkmark$ & $\checkmark$ & & $\checkmark$ & $\checkmark$ & $\checkmark$ & $\checkmark$ & & \\
\hline Baseline: Recite months & $\checkmark$ & $\checkmark$ & & $(\checkmark)$ & $(\checkmark)$ & & & & \\
\hline $\begin{array}{l}\text { Sentence generation vs } \\
\text { recite months }\end{array}$ & & & & $(\checkmark)$ & $(\checkmark)$ & $\checkmark$ & $\checkmark$ & & \\
\hline
\end{tabular}

$\checkmark=$ engaged

$(\checkmark)$ = sometimes engaged (e.g. depending on task demands, modality of stimuli, occurrence of automatic linguistic processing) 


\section{Table 4 (on next page)}

Language processes isolated by subtraction of different baseline tasks from semantic decision

Table shows comparison of active and passive baseline tasks as used for subtraction with semantic decision, in terms of the language and domain-general processes engaged by each paradigm and isolated by the subtraction contrast. Tick = engaged, bracketed tick = sometimes engaged (e.g. depending on task demands, modality of stimuli, occurence of automatic linguistic processing). 


\begin{tabular}{l|lcccc|ccc} 
& $\begin{array}{l}\text { Speech } \\
\text { motor } \\
\text { planning/ } \\
\text { articulation }\end{array}$ & $\begin{array}{l}\text { Phono- } \\
\text { logical } \\
\text { access }\end{array}$ & $\begin{array}{l}\text { Ortho- } \\
\text { graphical } \\
\text { processing }\end{array}$ & Semantics & $\begin{array}{l}\text { Word Syntax } \\
\text { retrieval }\end{array}$ & $\begin{array}{l}\text { Working } \\
\text { memory }\end{array}$ & $\begin{array}{l}\text { Motor } \\
\text { processing }\end{array}$ & $\begin{array}{l}\text { Auditory } \\
\text { processing }\end{array}$ \\
Contrast & & $(\checkmark)$ & $(\checkmark)$ & $\checkmark$ & $(\checkmark)$ & $\checkmark$ & $(\checkmark)$ \\
\hline $\begin{array}{l}\text { Task: Semantic decision } \\
\text { Baseline: Rest }\end{array}$ & & & $(\checkmark)$ & $(\checkmark)$ & $(\checkmark)$ & $(\checkmark)$ & $\checkmark$ & $(\checkmark)$ \\
\hline Semantic decision vs Rest & & $(\checkmark)$ & $\checkmark$ & $(\checkmark)$ & $\checkmark$ & $(\checkmark)$ & $(\checkmark)$ \\
\hline $\begin{array}{l}\text { Task: Semantic decision } \\
\text { Baseline: Perceptual } \\
\text { decision }\end{array}$ & & $(\checkmark)$ & $(\checkmark)$ & $\checkmark$ & $\checkmark$ & \\
\hline $\begin{array}{l}\text { Semantic vs Perceptual } \\
\text { decision }\end{array}$ & & & & & & & $(\checkmark)$ & \\
\hline
\end{tabular}

$\checkmark=$ engaged

$(\checkmark)$ = sometimes engaged (e.g. depending on task demands, modality of stimuli, occurrence of automatic linguistic processing) 